\title{
CONVERGENT AND ASYMPTOTIC EXPANSIONS OF SOLUTIONS OF DIFFERENTIAL EQUATIONS WITH A LARGE PARAMETER: OLVER CASES II AND III
}

\author{
CHELO FERREIRA, JOSÉ L. LÓPEZ AND ESTER PÉREZ SINUSÍA \\ Communicated by Bernd Silbermann
}

\begin{abstract}
We consider the asymptotic method designed by Olver [6] for linear differential equations of second order containing a large (asymptotic) parameter $\Lambda$, in particular, the second and third cases studied by Olver: differential equations with a turning point (second case) or a singular point (third case). It is well known that his method gives the Poincaré-type asymptotic expansion of two independent solutions of the equation in inverse powers of $\Lambda$. In this paper, we add initial conditions to the differential equation and consider the corresponding initial value problem. By using the Green's function of an auxiliary problem, we transform the initial value problem into a Volterra integral equation of the second kind. Then, using a fixed point theorem, we construct a sequence of functions that converges to the unique solution of the problem. This sequence also has the property of being an asymptotic expansion for large $\Lambda$ (not of Poincaré-type) of the solution of the problem. Moreover, we show that the technique also works for nonlinear differential equations with a large parameter.
\end{abstract}

1. Introduction. The most famous asymptotic method for second order linear differential equations containing a large parameter is, without any doubt, Olver's method [6, Chapters 10, 11, 12]. Olver divides the study into three canonical cases, say I, II and III (corresponding respectively to Chapters 10,11 and 12). In Case I, Olver completes

2010 AMS Mathematics subject classification. Primary 34A12, 34B27, 41A58, 41A60, 45D05.

Keywords and phrases. Second order differential equations, turning points, regular singular points, Volterra integral equations of the second kind, asymptotic expansions, Green functions, fixed point theorems, airy functions, Bessel functions.

The Dirección General de Ciencia y Tecnología (REF. MTM2010-21037) is acknowledged for its financial support.

Received by the editors on March 18, 2013, and in revised form on March 18, 2014. 
the theory developed in the well known Liouville-Green approximation, giving a rigorous meaning to the approximation and providing error bounds for the expansions of solutions of differential equations free of transition points [6, Chapter 10]. In Cases II and III, Olver generalizes the theory considering differential equations with a turning point or with a regular singular point, respectively.

Consider any homogeneous second order linear differential equation $\ddot{u}+p(t) \dot{u}+q(t) u=0$, where the dot means derivative with respect to the independent variable $t$ (real or complex). This variable $t$ ranges over a real interval or a complex domain, neither of which need to be bounded. After the change of unknown $u=w \cdot \exp \left[-(1 / 2) \int^{t} p(s) d s\right]$, this equation becomes the following equation for the new unknown $w$ : $\ddot{w}-Q(t) w=0$, where $Q(t):=(1 / 4) p^{2}+(1 / 2) \dot{p}-q[\mathbf{1 1}$, Chapter 4 , Section 5].

A more detailed version of the following discussion may be found in [6, Chapter 10, Section 1]. Suppose that $p$ and/or $q$ contain a (large) parameter $\Lambda$, real or complex, in such a way that $Q(t)$ may be written in the form $Q(t)=\Lambda^{2} \tilde{f}(t)+h(t)$ for certain functions $\widetilde{f}$ and $h$. Now, for an (at this moment) arbitrary function $t(x)$, we introduce a double change of both, the unknown $w$ and the independent variable $t: t \rightarrow x$ and $w \rightarrow y=\dot{x}^{1 / 2} w$. After this double (at this moment unspecified) change of variables, the differential equation for $w$ becomes the following differential equation for $y$ :

$$
y^{\prime \prime}=\left[\Lambda^{2}\left(\frac{d t}{d x}\right)^{2} f(x)+g(x)\right] y,
$$

where the prime denotes derivative with respect to $x, f(x):=\widetilde{f}(t(x))$, and

$$
\begin{aligned}
g(x) & :=\left(\frac{d t}{d x}\right)^{2} h(x)-\frac{1}{2}\{t(x), x\} \\
& =\left(\frac{d t}{d x}\right)^{2} h(x)+\left(\frac{d t}{d x}\right)^{1 / 2} \frac{d^{2}}{d x^{2}}\left(\frac{d t}{d x}\right)^{-1 / 2} .
\end{aligned}
$$

The precise transformation $t(x)$ is now fixed by specifying the relation between $t$ and $x$ in such a manner that (i) $t$ and $x$ are analytic functions of each other at the transition point of the differential equation (if any) and (ii) the approximating differential equation obtained by neglecting 
all or part of $g(x)$ has solutions which are functions of a single variable. The choices are:

Case I. $\left(\frac{d t}{d x}\right)^{2} f(x)=1$, which means $x=\int f^{1 / 2}(t) d t$.

Case II. $\left(\frac{d t}{d x}\right)^{2} f(x)=x$, which means $\frac{2}{3} x^{3 / 2}=\int_{t_{0}}^{t} f^{1 / 2}(s) d s$.

Case III. $\left(\frac{d t}{d x}\right)^{2} f(x)=1 / x$, which means $2 x^{1 / 2}=\int_{t_{0}}^{t} f^{1 / 2}(s) d s$.

Then, equation (1) reduces to

$$
y^{\prime \prime}=\left[\Lambda^{2} x^{m}+g(x)\right] y,
$$

with $m=0$ (Case I), $m=1$ (Case II) or $m=-1$ (Case III). In [4] we give an alternative approximation to Olver's expansion for Case I that is not only asymptotic, but also convergent. In this paper, we pursue the same objective for Cases II and III. In the remainder of this introduction we briefly summarize Olver's method in Cases II and III.

1.1. Case II. When $\Lambda$ is large, it is reasonable to neglect $g(x)$ in

$$
y^{\prime \prime}=\left[\Lambda^{2} x+g(x)\right] y,
$$

to get a first order approximation of the solutions of this differential equation: any linear combination of the Airy functions $\mathrm{Ai}\left(\Lambda^{2 / 3} x\right)$ and $\operatorname{Bi}\left(\Lambda^{2 / 3} x\right)$. Then, it is reasonable to seek for formal solutions of (3) of the form

$$
\begin{aligned}
& y_{1}(x) \sim \operatorname{Ai}\left(\Lambda^{2 / 3} x\right) \sum_{n=0}^{\infty} \frac{A_{n}(x)}{\Lambda^{n}}, \\
& y_{2}(x) \sim \operatorname{Bi}\left(\Lambda^{2 / 3} x\right) \sum_{n=0}^{\infty} \frac{B_{n}(x)}{\Lambda^{n}} .
\end{aligned}
$$

As Olver shows in [6, Chapter 11, Section 7], this first naive attempt does not work and one must try instead a formal solution of the form

$$
y_{1}(x) \sim \operatorname{Ai}\left(\Lambda^{2 / 3} x\right) \sum_{n=0}^{\infty} \frac{A_{n}(x)}{\Lambda^{2 n}}+\frac{\operatorname{Ai}^{\prime}\left(\Lambda^{2 / 3} x\right)}{\Lambda^{4 / 3}} \sum_{n=0}^{\infty} \frac{B_{n}(x)}{\Lambda^{2 n}} .
$$

Introducing this expansion in (3) and equating coefficients with equal powers of $\Lambda$, we see that the differential equation is satisfied, formally, 
if, for $n=0,1,2, \ldots$,

$$
\left\{\begin{array}{l}
A_{n}^{\prime \prime}(x)-g(x) A_{n}(x)+2 x B_{n}^{\prime}(x)+B_{n}(x)=0, \\
2 A_{n+1}^{\prime}(x)+B_{n}^{\prime \prime}(x)-g(x) B_{n}(x)=0
\end{array}\right.
$$

with $A_{0}(x)$ an arbitrary constant (we may take $A_{0}(x)=1$ without loss of generality). Then, higher coefficients are determined recursively by

$$
\begin{aligned}
A_{n+1}(x) & =-\frac{1}{2} B_{n}^{\prime}(x)+\frac{1}{2} \int g(x) B_{n}(x) d x, \quad n=0,1,2, \ldots, \\
B_{n}(x) & =\frac{1}{2 \sqrt{x}} \int_{0}^{x}\left[g(t) A_{n}(t)-A_{n}^{\prime \prime}(t)\right] \frac{d t}{\sqrt{t}}, \quad n=0,1,2, \ldots,
\end{aligned}
$$

the constants of integration being arbitrary. A second formal solution may be obtained from (4) by replacing $\mathrm{Ai}(x)$ by $\mathrm{Bi}(x)$ :

$$
y_{2}(x) \sim \operatorname{Bi}\left(\Lambda^{2 / 3} x\right) \sum_{n=0}^{\infty} \frac{A_{n}(x)}{\Lambda^{2 n}}+\frac{\operatorname{Bi}^{\prime}\left(\Lambda^{2 / 3} x\right)}{\Lambda^{4 / 3}} \sum_{n=0}^{\infty} \frac{B_{n}(x)}{\Lambda^{2 n}} .
$$

In general, expansions (4) and (6) are divergent. However, when they are properly interpreted, they give good approximations of the solutions of (3). The important contribution of Olver's theory is the proof of the asymptotic character of expansions (4) and (6). More precisely, suppose that $g(x)$ is analytic in a certain region of the complex plane. Then, both (4) and (6), with the coefficients $A_{n}(x)$ and $B_{n}(x)$ given in (5), are asymptotic expansions for large $\Lambda$ of two independent solutions $y_{1}(x)$ and $y_{2}(x)$ of (3) [6, page 410, Theorem 7.1]. Moreover, in that theorem, we can also find error bounds for the remainders of the expansions:

$$
R_{n, 1}(x):=y_{1}(x)-\left[\operatorname{Ai}\left(\Lambda^{2 / 3} x\right) \sum_{k=0}^{n} \frac{A_{k}(x)}{\Lambda^{2 k}}+\frac{\operatorname{Ai}^{\prime}\left(\Lambda^{2 / 3} x\right)}{\Lambda^{4 / 3}} \sum_{k=0}^{n-1} \frac{B_{k}(x)}{\Lambda^{2 k}}\right]
$$

and

$$
R_{n, 2}(x):=y_{2}(x)-\left[\operatorname{Bi}\left(\Lambda^{2 / 3} x\right) \sum_{k=0}^{n} \frac{A_{k}(x)}{\Lambda^{2 k}}+\frac{\operatorname{Bi}^{\prime}\left(\Lambda^{2 / 3} x\right)}{\Lambda^{4 / 3}} \sum_{k=0}^{n-1} \frac{B_{k}(x)}{\Lambda^{2 k}}\right] .
$$

In [6, Chapter 11] we can also find several properties of the expansions (4) and (6), like uniformity properties and examples of special functions. Discussions about the regions of validity of the expansions and 
other more general equations, in particular inhomogeneous equations, are also presented in [6, Chapter 11].

1.2. Case III. As in Case II, when $\Lambda$ is large, it is reasonable to neglect $g(x)$ in

$$
y^{\prime \prime}=\left[\frac{\Lambda^{2}}{x}+g(x)\right] y,
$$

to get a first order approximation of the solutions of this differential equation: Bessel functions (or modified Bessel functions), of order 1. Then, we seek for formal solutions of (7) of the form

$$
\begin{aligned}
& y_{1}(x) \sim \sqrt{x} I_{1}(\Lambda \sqrt{x}) \sum_{n=0}^{\infty} \frac{A_{n}(x)}{\Lambda^{2 n}}+\frac{x}{\Lambda} I_{2}(\Lambda \sqrt{x}) \sum_{n=0}^{\infty} \frac{B_{n}(x)}{\Lambda^{2 n}}, \\
& y_{2}(x) \sim \sqrt{x} K_{1}(\Lambda \sqrt{x}) \sum_{n=0}^{\infty} \frac{A_{n}(x)}{\Lambda^{2 n}}+\frac{x}{\Lambda} K_{2}(\Lambda \sqrt{x}) \sum_{n=0}^{\infty} \frac{B_{n}(x)}{\Lambda^{2 n}} .
\end{aligned}
$$

The coefficients $A_{n}(x)$ and $B_{n}(x)$ are obtained from a system of recurrences similar to (5) (see [6, Chapter 12] for details).

Once again, in general, expansions (8) and (9) are divergent, and the contribution of Olver's theory is the proof of the asymptotic character of these expansions.

Expansions (4), (6), (8) and (9) are asymptotic expansions of solutions of (3) and (7), but in general, they are divergent. The purpose of this paper is to design a method that approximates one solution of (3) and of (7) by means of an asymptotic series that is also convergent. Without any extra effort, we may consider a nonlinear generalization of equations (3) and (7).

In the next section, we consider initial value problems associated to a nonlinear generalization of equations (3) and (7), respectively. By using the fixed point theorem of Banach and the Green's function of an auxiliary initial value problem, we obtain uniformly convergent expansions of that solution in terms of iterated integrals of $\mathrm{Ai}(x)$ and $\mathrm{Bi}(x)$ (Case II), and iterated integrals of $I_{1}(x)$ and $K_{1}(x)$ (Case III). In Section 3 we show that these expansions are asymptotic expansions for large $\Lambda$ of the unique solution of the initial value problem. In Section 4, we particularize our theory to linear equations, in order to compare 
with Olver's expansions. Some examples are presented in Section 5. Section 6 contains a few remarks and conclusions.

\section{Nonlinear initial value problems.}

2.1. Case II. Consider the following nonlinear initial value problem

$$
\left\{\begin{array}{l}
y^{\prime \prime}-\Lambda^{3} x y=f(x, y) \quad \text { in }[-X, X], \\
y(0)=y_{0}, \quad y^{\prime}(0)=y_{0}^{\prime},
\end{array}\right.
$$

where the function $f:[-X, X] \times \mathbf{C} \rightarrow \mathbf{C}$ is continuous in its two variables, $X>0, y_{0}, y_{0}^{\prime}, \Lambda \in \mathbf{C}, y_{0}=\mathcal{O}(1)$ and $y_{0}^{\prime}=\mathcal{O}(\Lambda)$ as $\Lambda \rightarrow \infty$. Without loss of generality, we restrict ourselves to $\Re \Lambda \geq 0$. In the following analysis we require for $f$ to satisfy a Lipschitz condition in its second variable:

$$
|f(x, y)-f(x, z)| \leq K|y-z|, \quad \text { for all } y, z \in \mathbf{C} \quad \text { and } x \in[-X, X]
$$

with $K$ a positive constant independent of $x, y, z$. Also, it is convenient to define the following function:

$$
\widetilde{\operatorname{Bi}}(z):=1+|\operatorname{Bi}(z)|, \quad \text { for } z \in \mathbf{C} .
$$

Theorem 2.1. Let $f:[-X, X] \times \mathbf{C} \rightarrow \mathbf{C}$ be continuous and satisfy (11). Then, problem (10) has a unique solution $y(x)$. Moreover,

(i) For $n=0,1,2, \ldots$, and $y_{0}(x)=\phi(x)$, the sequence

$$
\begin{aligned}
& \left\{\begin{array}{l}
y_{0}(x)=\phi(x):=\pi\left\{\left[y_{0} \operatorname{Bi}^{\prime}(0)-\frac{y_{0}^{\prime}}{\Lambda} \operatorname{Bi}(0)\right] \operatorname{Ai}(\Lambda x)\right. \\
\left.\quad-\left[y_{0} \operatorname{Ai}^{\prime}(0)-\frac{y_{0}^{\prime}}{\Lambda} \operatorname{Ai}(0)\right] \operatorname{Bi}(\Lambda x)\right\}, \\
y_{n+1}(x)=\phi(x)+\frac{\pi}{\Lambda} \int_{0}^{x}[\operatorname{Bi}(\Lambda x) \operatorname{Ai}(\Lambda t)-\operatorname{Ai}(\Lambda x) \operatorname{Bi}(\Lambda t)] f\left(t, y_{n}(t)\right) d t,
\end{array}\right. \\
& \text { converges to } y(x) \text { uniformly for } x \in[-X, X] \text {. }
\end{aligned}
$$

$$
R_{n}(x):=\widetilde{\mathrm{Bi}}^{-1}(x)\left[y(x)-y_{n}(x)\right]
$$

is bounded by

$$
\left|R_{n}(x)\right| \leq \frac{K^{n}|C x|^{n}}{|\Lambda|^{n} n !}\left\|\widetilde{\mathrm{Bi}}^{-1}(\Lambda \cdot)(y-\phi)\right\|_{\infty},
$$


where $C$ is a positive constant independent of $\Lambda$ and $x$ ( $a$ uniform bound for the kernel given below).

Proof. Using the Wronskian of the Airy functions is $\{\mathrm{Ai}(x), \mathrm{Bi}(x)\}$ $=1 / \pi$, we can see that the function $\phi(x)$ defined in (13) is the unique solution of the auxiliary initial value problem

$$
\left\{\begin{array}{l}
\phi^{\prime \prime}-\Lambda^{3} x \phi=0 \quad \text { in }[-X, X] \\
\phi(0)=y_{0}, \quad \phi^{\prime}(0)=y_{0}^{\prime}
\end{array}\right.
$$

Then, after the change of unknown $y(x) \rightarrow u(x):=y(x)-\phi(x)$, problem (10) reads

$$
\left\{\begin{array}{l}
u^{\prime \prime}-\Lambda^{3} x u=F(x, u):=f(x, u+\phi) \quad \text { in }[-X, X] \\
u(0)=u^{\prime}(0)=0
\end{array}\right.
$$

We seek for solutions of the equation $\mathbf{L}[u]:=u^{\prime \prime}-\Lambda^{3} x u-F(x, u)=0$ in the Banach space $\mathcal{B}=\{u:[-X, X] \rightarrow \mathbf{C}, u \in \mathcal{C}[-X, X]\}$ equipped with the norm

$$
\|u\|_{\infty}=\sup _{x \in[-X, X]}|u(x)|
$$

Then, we solve the equation $\mathbf{L}[u]=0$ for $u$ by using Green's function of the operator $\mathbf{M}[u]:=u^{\prime \prime}-\Lambda^{3} x u$ accompanied by homogeneous initial conditions [10]. That is, $G(x, t)$ is the unique solution of the initial value problem

$$
\left\{\begin{array}{l}
G_{x x}(x, t)-\Lambda^{3} x G(x, t)=\delta(x-t) \quad \text { in }(x, t) \in[-X, X]^{2}, \\
G(0, t)=G_{x}(0, t)=0 .
\end{array}\right.
$$

After a straightforward computation, we obtain

$$
G(x, t)=\frac{\pi}{\Lambda}[\operatorname{Ai}(\Lambda t) \operatorname{Bi}(\Lambda x)-\operatorname{Bi}(\Lambda t) \operatorname{Ai}(\Lambda x)] \chi_{[0, x]}(t),
$$

where $\chi_{[0, x]}(t)$ is the characteristic function of the interval $[0, x]$ for $x>0$ and of the interval $[x, 0]$ for $x<0$. Then, any solution $u(x)$ of (16) is a solution of the Volterra integral equation of the second kind:

$$
u(x)=\frac{\pi}{\Lambda} \int_{0}^{x}[\operatorname{Ai}(\Lambda t) \operatorname{Bi}(\Lambda x)-\operatorname{Bi}(\Lambda t) \operatorname{Ai}(\Lambda x)] f(t, u(t)+\phi(t)) d t .
$$


Or equivalently, defining

$$
\widetilde{u}(x):=\widetilde{\mathrm{Bi}}^{-1}(\Lambda x) u(x), \quad \widetilde{\phi}(x):=\widetilde{\mathrm{Bi}}^{-1}(\Lambda x) \phi(x),
$$

we have that, for any solution $u(x)$ of $(16), \widetilde{u}(x)$ is a solution of the Volterra integral equation of the second kind

$$
\widetilde{u}(x)=[\mathbf{T} \widetilde{u}](x),
$$

where we have defined the operator $\mathbf{T}$ in the form

$$
[\mathbf{T} \widetilde{u}](x):=\frac{1}{\Lambda} \int_{0}^{x} K_{\Lambda}(x, t) f(t, \widetilde{u}(t)+\widetilde{\phi}(t)) d t,
$$

with

$$
K_{\Lambda}(x, t):=\pi \frac{\widetilde{\operatorname{Bi}}(\Lambda t)}{\widetilde{\operatorname{Bi}}(\Lambda x)}[\operatorname{Ai}(\Lambda t) \operatorname{Bi}(\Lambda x)-\operatorname{Bi}(\Lambda t) \operatorname{Ai}(\Lambda x)] .
$$

Airy functions are continuous functions in the whole complex plane and $\widetilde{\operatorname{Bi}}(z) \neq 0$ for all $z \in \mathbf{C}$. Using, in addition, the asymptotic behavior of these functions [ 5 , subsections 9.7 (i), 9.7 (ii)], we find that $\left|K_{\Lambda}(x, t)\right| \leq C$ for a certain positive constant $C$ independent of $\Lambda \in \mathbf{C}$ and $x, t \in[-X, X]$ with $0 \leq t / x \leq 1$. Using this bound, from $[\mathbf{1 0}$, Chapter 4, equation (4.13)], we find that, for any couple $z, w \in \mathcal{B}$ and $x \in[-X, X]$,

$$
\left|\left[\mathbf{T}^{n} z\right](x)-\left[\mathbf{T}^{n} w\right](x)\right| \leq \frac{K^{n}}{n !}\left|\frac{C x}{\Lambda}\right|^{n}\|z-w\|_{\infty}, \quad n=1,2,3, \ldots
$$

Then [10, Chap 4. eq. (4.14)],

$$
\left\|\mathbf{T}^{n} z-\mathbf{T}^{n} w\right\|_{\infty} \leq \frac{K^{n}}{n !}\left|\frac{C X}{\Lambda}\right|^{n}\|z-w\|_{\infty}, \quad n=1,2,3, \ldots .
$$

This means that the operator $\mathbf{T}^{n}$ is contractive in $\mathcal{B}$ for large enough $n$ and the successive approximations $\widetilde{u}_{n+1}=\mathbf{T}\left(\widetilde{u}_{n}\right), n=0,1,2, \ldots$, $\widetilde{u}_{0}(x)=0$, converge uniformly in $x \in[-X, X]$ to $\widetilde{u}(x)$ [10, Chapter 4 , Section 4]. In other words, $u(x)=\widetilde{\operatorname{Bi}}(\Lambda x) \widetilde{u}(x)$ is the unique solution of (16). Or, equivalently, the sequence $y_{n}(x)=\widetilde{\mathrm{Bi}}(\Lambda x)\left[\widetilde{u}_{n}(x)+\widetilde{\phi}(x)\right]$ given in (13) converges uniformly in $x \in[-X, X]$ to the unique solution of (10). This proves thesis (i). 
To prove thesis (ii), we set $z=\widetilde{u}$ and $w=\widetilde{u}_{0}=0$ in (23). Using that $\mathbf{T}^{n} \widetilde{u}=\widetilde{u}$ and $\mathbf{T}^{n} \widetilde{u}_{0}=\widetilde{u}_{n}$, we find

$$
\left|\widetilde{u}(x)-\widetilde{u}_{n}(x)\right| \leq K^{n} \frac{1}{n !}\left|\frac{C x}{\Lambda}\right|^{n}\|\widetilde{u}\|_{\infty} .
$$

Using $y(x)=\widetilde{\mathrm{Bi}}(\Lambda x) \widetilde{u}(x)+\phi(x)$ and $y_{n}(x)=\widetilde{\mathrm{Bi}}(\Lambda x) \widetilde{u}_{n}(x)+\phi(x)$ in (25), we find (15).

Remark 2.2. Of course, the existence and uniqueness of the solution of problem (10) is not new but a direct consequence of Picard-Lindelöf's theorem [3, Chapter 8, Section 2]. In fact, the proof of PicardLindelöf's theorem using Picard's iteration is similar to the above derivation. The unique difference is the choice of the "main operator" $\mathbf{M}[u]$ that, in the standard Picard-Lindelöf theorem, is chosen to be $\mathbf{M}[u]=u^{\prime \prime}$. The advantage of taking $\mathbf{M}[u]=u^{\prime \prime}-\Lambda^{3} x u$, instead of $\mathbf{M}[u]=u^{\prime \prime}$, is that, for large $\Lambda$, we have a faster convergence of the iteration (13) than the standard Picard's iteration, as long as the operator $\mathbf{M}[u]=u^{\prime \prime}-\Lambda^{3} x u$ is "closer" to $\mathbf{L}[u]$ than the operator $\mathbf{M}[u]=u^{\prime \prime}$ : the error bound for the standard Picard's iteration is similar to (15) but replacing $K$ by $K+|\Lambda|^{3} X$. When $\Lambda$ is large compared with $K$, we have that (13) converges faster than the standard Picard's iteration. Moreover, in the next section, we show that the recurrence $y_{n}(x)$ given in (13) is not only convergent (to the unique solution $y(x)$ of (10)), but also an asymptotic expansion of $y(x)$ for large $\Lambda$.

2.2. Case III. Consider the following nonlinear initial value problem

$$
\left\{\begin{array}{l}
y^{\prime \prime}-\frac{\Lambda^{2}}{x} y=f(x, y) \quad \text { in }[-X, X], \\
y^{\prime}(0)=y_{0}^{\prime}
\end{array}\right.
$$

where the function $f:[-X, X] \times \mathbf{C} \rightarrow \mathbf{C}$ is continuous in its two variables and satisfies (11). We consider $X>0, y_{0}^{\prime}, \Lambda \in \mathbf{C}$ with $\Re \Lambda \geq 0$, and $y_{0}^{\prime}=\mathcal{O}(\Lambda)$ as $\Lambda \rightarrow \infty$. We also need to define the function

$$
\widetilde{I}_{1}(z):=1+\left|I_{1}(2 \sqrt{z})\right|, \quad \text { for } z \in \mathbf{C} .
$$

We have the following theorem. 
Theorem 2.3. Let $f:[-X, X] \times \mathbf{C} \rightarrow \mathbf{C}$ be continuous and satisfy (11). Then, problem (26) has a unique solution $y(x)$. Moreover:

(i) For $n=0,1,2, \ldots$, the sequence

$$
\left\{\begin{array}{l}
y_{0}(x)=\phi(x):=\frac{y_{0}^{\prime}}{\Lambda} \sqrt{x} I_{1}(2 \Lambda \sqrt{x}) \\
y_{n+1}(x)=\phi(x)+2 \int_{0}^{x} \sqrt{x t}\left[I_{1}(2 \Lambda \sqrt{x}) K_{1}(2 \Lambda \sqrt{t})\right. \\
\left.\quad-K_{1}(2 \Lambda \sqrt{x}) I_{1}(2 \Lambda \sqrt{t})\right] f\left(t, y_{n}(t)\right) d t
\end{array}\right.
$$

converges to $y(x)$ uniformly for $x \in[-X, X]$.

(ii) The remainder

$$
R_{n}(x):={\widetilde{I_{1}}}^{-1}\left(\Lambda^{2} x\right)\left[y(x)-y_{n}(x)\right]
$$

is bounded by

$$
\left|R_{n}(x)\right| \leq \frac{K^{n}}{n !}\left|\frac{C x}{\Lambda}\right|^{n}\left\|\widetilde{I}_{1}\left(\Lambda^{2} \cdot\right)(y-\phi)\right\|_{\infty},
$$

where $C$ is a positive constant independent of $\Lambda$ and $x$ ( $a$ uniform bound for the kernel given below).

Proof. Similar to the proof of Theorem 1, we replace the kernel (22) by:

$$
K_{\Lambda}(x, t):=2 \Lambda \sqrt{x t} \frac{\widetilde{I_{1}}\left(\Lambda^{2} t\right)}{\widetilde{I}_{1}\left(\Lambda^{2} x\right)}\left[K_{1}(2 \Lambda \sqrt{t}) I_{1}(2 \Lambda \sqrt{x})-I_{1}(2 \Lambda \sqrt{t}) K_{1}(2 \Lambda \sqrt{x})\right] .
$$

The Bessel functions $I_{\nu}(z)$ and $K_{\nu}(z)$ are continuous functions of $z$ in $\mathbf{C} \backslash(-\infty, 0]$ and $\widetilde{I}_{1}(z) \neq 0$ for all $z \in \mathbf{C}$. Using, in addition, the asymptotic behavior of these functions at $z=0$ [5, equations (10.25.2), $(10.27 .4),(10.27 .5)]$ and at $z=\infty[\mathbf{5}$, subsection 10.40 (i)], we find that $\left|K_{\Lambda}(x, t)\right| \leq C$ for a certain positive constant $C$ independent of $\Lambda \in \mathbf{C}$ and $x, t \in[-X, X]$ with $0 \leq t / x \leq 1$.

Remark 2.4. The existence and uniqueness of the solution of (26) is a new result, as Picard-Lindelöf's theorem does not apply to this problem. Then, Theorem 2.3 is a kind of Picard-Lindelöf theorem for second order differential equations with initial datum given at the regular singular point of the equation. It is worth noting that, as a difference from the regular problem, only one initial datum is required: 
the value of the derivative at the regular singular point. It is natural to also consider problem (26), but to replace the condition $y^{\prime}(0)=y_{0}^{\prime}$ by the condition $y(0)=y_{0}$. Or, consider problem (26) with both conditions: $y(0)=y_{0}$ and $y^{\prime}(0)=y_{0}^{\prime}$. In general, neither of these two initial value problems has a unique solution (and this is why we have not considered them here). For example, the problem

$$
\left\{\begin{array}{l}
y^{\prime \prime}-\frac{\Lambda^{2}}{x} y=0 \quad \text { in }[-X, X] \\
y(0)=0
\end{array}\right.
$$

has an infinite number of solutions: $y(x)=c \sqrt{x} I_{1}(2 \Lambda \sqrt{x})$, with $c$ an arbitrary constant. On the other hand, the problem

$$
\left\{\begin{array}{l}
y^{\prime \prime}-\frac{\Lambda^{2}}{x} y=0 \quad \text { in }[-X, X] \\
y(0)=1, \quad y^{\prime}(0)=0
\end{array}\right.
$$

has no solution.

\section{Asymptotic properties of the expansions.}

3.1. Case II. We have seen in Theorem 1 that the unique solution $y(x)$ of problem (10) may be obtained from the limit $y(x)=$ $\lim _{n \rightarrow \infty} y_{n}(x)$ uniformly in $[-X, X]$, where $y_{n}(x)$ is defined by the recurrence (13). In other words, $y(x)$ admits the series expansion

$$
\begin{aligned}
y(x) & =\phi(x)+\sum_{k=0}^{\infty}\left[y_{k+1}(x)-y_{k}(x)\right] \\
& =\phi(x)+\widetilde{\operatorname{Bi}}(\Lambda x) \sum_{k=0}^{\infty}\left[\widetilde{u}_{k+1}(x)-\widetilde{u}_{k}(x)\right],
\end{aligned}
$$

with

$$
\widetilde{u}_{n}(x):=\widetilde{\mathrm{Bi}}^{-1}(\Lambda x)\left[y_{n}(x)-\phi(x)\right], \quad n=0,1,2, \ldots .
$$

Using (14), we may write (31) in the form

$$
y(x)=\phi(x)+\sum_{k=0}^{n-1}\left[y_{k+1}(x)-y_{k}(x)\right]+\widetilde{\operatorname{Bi}}(\Lambda x) R_{n}(x)
$$




$$
=\phi(x)+\widetilde{\operatorname{Bi}}(\Lambda x)\left[\sum_{k=0}^{n-1}\left[\widetilde{u}_{k+1}(x)-\widetilde{u}_{k}(x)\right]+R_{n}(x)\right] .
$$

Then, we have the following theorem.

Theorem 3.1. Let the functions $y_{n}(x)$ be defined by the recurrence (13), where $f:[-X, X] \times \mathbf{C} \rightarrow \mathbf{C}$ is continuous in $[-X, X]$ and satisfies (11). Then, the expansion (33) is an asymptotic expansion for large $\Lambda$ of the unique solution of (10), uniformly for $x \in[-X, X]$. More precisely, for $n=1,2,3, \ldots$,

$$
\left\|\widetilde{u}_{n}(x)-\widetilde{u}_{n-1}(x)\right\|_{\infty}=\mathcal{O}\left(|\Lambda|^{-n}\right)
$$

and

$$
\left\|R_{n}(x)\right\|_{\infty}=\mathcal{O}\left(|\Lambda|^{-n-1}\right)
$$

Proof. From definition (21), we have

$$
\widetilde{u}_{n}(x)=\left[\mathbf{T} \widetilde{u}_{n-1}\right](x)=\frac{1}{\Lambda} \int_{0}^{x} K_{\Lambda}(x, t) f\left(t, \widetilde{u}_{n-1}(t)+\widetilde{\phi}(t)\right) d t
$$

and

$$
\widetilde{u}_{n+1}(x)=\left[\mathbf{T} \widetilde{u}_{n}\right](x)=\frac{1}{\Lambda} \int_{0}^{x} K_{\Lambda}(x, t) f\left(t, \widetilde{u}_{n}(t)+\widetilde{\phi}(t)\right) d t .
$$

with $K_{\Lambda}(x, t)$ defined in (22). Subtracting (36) from (35) and using the bounds $\left|K_{\Lambda}(x, t)\right| \leq C$ and (11), we find that

$$
\left\|\widetilde{u}_{n+1}-\widetilde{u}_{n}\right\|_{\infty} \leq\left|\frac{X}{\Lambda}\right| C K\left\|\widetilde{u}_{n}-\widetilde{u}_{n-1}\right\|_{\infty} .
$$

We have $\widetilde{u}_{0}(x)=0$ and $\widetilde{u}_{1}(x)=\left[\mathbf{T} \widetilde{u}_{0}\right](x)=\mathcal{O}\left(|\Lambda|^{-1}\right)$ uniformly for $x \in[-X, X]$. Using this and (37), the first thesis in (34) follows by induction over $n$.

Observe that $\widetilde{u}=\lim _{n \rightarrow \infty} \widetilde{u}_{n}=\sum_{k=0}^{\infty}\left[\widetilde{u}_{k+1}-\widetilde{u}_{k}\right]=\sum_{k=0}^{\infty} \mathcal{O}\left(|\Lambda|^{-k-1}\right)$ $=\mathcal{O}\left(|\Lambda|^{-1}\right)$ uniformly for $x \in[-X, X]$. This and inequality (25) prove the second thesis in (34). 
3.2. Case III. We have seen in Theorem 2 that the unique solution $y(x)$ of problem (26) may be obtained from the limit $y(x)=$ $\lim _{n \rightarrow \infty} y_{n}(x)$ uniformly in $[-X, X]$, where $y_{n}(x)$ is the sequence defined in (27). In other words, and analogously to Case II, in this Case III, $y(x)$ admits the series expansion

$$
y(x)=\phi(x)+\widetilde{I}_{1}\left(\Lambda^{2} x\right)\left[\sum_{k=0}^{n-1}\left[\widetilde{u}_{k+1}(x)-\widetilde{u}_{k}(x)\right]+R_{n}(x)\right]
$$

with

$$
\widetilde{u}_{n}(x):=\widetilde{I}_{1}^{-1}\left(\Lambda^{2} x\right)\left[y_{n}(x)-\phi(x)\right], \quad n=0,1,2, \ldots .
$$

And, we have the following theorem.

Theorem 3.2. Let the functions $y_{n}(x)$ be defined by the recurrence (27), where $f:[-X, X] \times \mathbf{C} \rightarrow \mathbf{C}$ is continuous in $[-X, X]$ and satisfies (11). Then, expansion (38) is an asymptotic expansion for large $\Lambda$ of the unique solution of (26), uniformly for $x \in[-X, X]$. More precisely, for $n=1,2,3, \ldots$,

$$
\left\|\widetilde{u}_{n}(x)-\widetilde{u}_{n-1}(x)\right\|_{\infty}=\mathcal{O}\left(|\Lambda|^{-n}\right)
$$

and

$$
\left\|R_{n}(x)\right\|_{\infty}=\mathcal{O}\left(|\Lambda|^{-n-1}\right) .
$$

Proof. Analogous to the proof of Theorem 3.1.

Example 3.3. Consider a mathematical pendulum [12, Chapter 5, Example 5.2] with a reaction term vanishing at the origin. Suppose that, at time $x=0$, the pendulum is at rest at the angular position $y=y_{0}$. Then, the angular position $y(x)$ is the unique solution of the initial value problem

$$
\left\{\begin{array}{l}
y^{\prime \prime}-\Lambda^{3} x y=\sin y, \quad x \in[-X, X] \\
y(0)=y_{0}, \quad y^{\prime}(0)=0 .
\end{array}\right.
$$

This problem is of the form considered in Theorem 2.1 with $f(x, y)=$ $\sin y$, and then $|f(x, y)-f(x, z)| \leq|y-z|$. This function is Lipschitz continuous for all $y, z \in \mathbf{C}$ with a Lipschitz constant $L=1$. From 

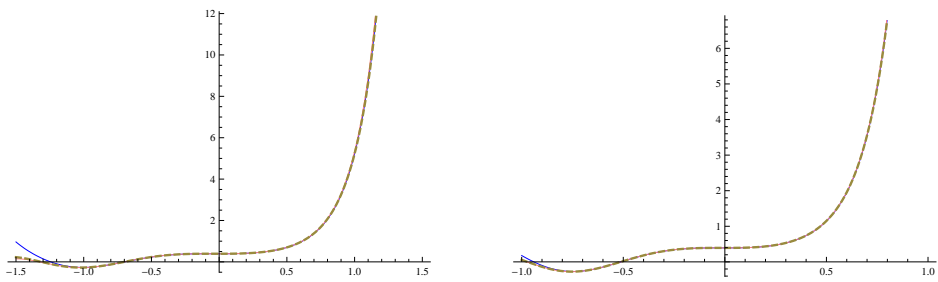

FIGURE 1. These graphs contain the exact solution (computed with a numerical integrator) $y(x)$ (dashed) and the approximations $y_{1}(x)$ (blue) and $y_{2}(x)$ (pink) for $\Lambda=3$ (left picture) and $\Lambda=4$ (right picture) with $y_{0}=\pi / 8$.

Theorem 2.1, we have that, for $n=0,1,2, \ldots$,

$$
\begin{gathered}
y_{0}(x)=\phi(x):=\pi y_{0}\left(\operatorname{Bi}^{\prime}(0) \operatorname{Ai}(\Lambda x)-\operatorname{Ai}^{\prime}(0) \operatorname{Bi}(\Lambda x)\right), \\
y_{n+1}(x)=\phi(x)+\frac{\pi}{\Lambda} \operatorname{Bi}(\Lambda x) \int_{0}^{x} \operatorname{Ai}(\Lambda t) \sin y_{n}(t) d t \\
-\frac{\pi}{\Lambda} \operatorname{Ai}(\Lambda x) \int_{0}^{x} \operatorname{Bi}(\Lambda t) \sin y_{n}(t) d t
\end{gathered}
$$

This sequence converges uniformly and absolutely to the unique solution of (41). Figure 1 illustrates the approximation of the unique solution of (41) supplied by this approximation.

4. The linear case. For a better comparison with Olver's method, we briefly indicate how the previous results look in the linear case: consider the initial value problems (10) and (26), with $f(x, y)=g(x) y$, where we only require the function $g:[-X, X] \rightarrow \mathbf{C}$ to be continuous. Then, Theorems $1,2,3$ and 4 hold, replacing $f(x, y)$ by $g(x) y$ and the positive constant $K$ by $\|g\|_{\infty}$.

\subsection{Examples.}

Example 4.1. For any $\Lambda \in \mathbf{C}$ and $X>0$, the unique solution of the initial value problem

$$
\left\{\begin{array}{l}
y^{\prime \prime}-\left(\Lambda^{3} x+\frac{x^{2}}{4}\right) y=0 \quad \text { in }[-X, X] \\
y(0)=1, \quad y^{\prime}(0)=U^{\prime}\left(-\Lambda^{6}, 2 \Lambda^{3}\right) / U\left(-\Lambda^{6}, 2 \Lambda^{3}\right)
\end{array}\right.
$$

is the normalized parabolic cylinder function $\widetilde{U}\left(-\Lambda^{6}, x+2 \Lambda^{3}\right):=$ $U\left(-\Lambda^{6}, x+2 \Lambda^{3}\right) / U\left(-\Lambda^{6}, 2 \Lambda^{3}\right)$. For this problem $g(x)=x^{2} / 4$ and, from 
Theorem 2.1 with $f(x, y)=g(x) y$, we obtain that, for $n=0,1,2, \ldots$, the sequence

$$
\left\{\begin{array}{c}
y_{0}(x)=\phi(x):=\pi\left\{\left[\mathrm{Bi}^{\prime}(0)-\frac{U^{\prime}\left(-\Lambda^{6}, 2 \Lambda^{3}\right)}{\Lambda U\left(-\Lambda^{6}, 2 \Lambda^{3}\right)} \mathrm{Bi}(0)\right] \mathrm{Ai}(\Lambda x)\right. \\
\left.-\left[\operatorname{Ai}^{\prime}(0)-\frac{U^{\prime}\left(-\Lambda^{6}, 2 \Lambda^{3}\right)}{\Lambda U\left(-\Lambda^{6}, 2 \Lambda^{3}\right)} \mathrm{Ai}(0)\right] \operatorname{Bi}(\Lambda x)\right\} \\
y_{n+1}(x)=\phi(x)+\frac{\pi}{4 \Lambda} \operatorname{Bi}(\Lambda x) \int_{0}^{x} \operatorname{Ai}(\Lambda t) t^{2} y_{n}(t) d t \\
-\frac{\pi}{4 \Lambda} \operatorname{Ai}(\Lambda x) \int_{0}^{x} \operatorname{Bi}(\Lambda t) t^{2} y_{n}(t) d t
\end{array}\right.
$$

converges uniformly for $x \in[-X, X]$, to the exact solution $\widetilde{U}\left(-\Lambda^{6}, x+\right.$ $\left.2 \Lambda^{3}\right)$. From Theorem 3.1 with $f(x, y)=g(x) y$, we know that it is also an asymptotic expansion of $\widetilde{U}\left(-\Lambda^{6}, x+2 \Lambda^{3}\right)$ for $\Lambda \rightarrow \infty$. Table 1 shows some numerical results for the approximation supplied by the recursion (42) compared with the approximation supplied by Olver's algorithm.

Example 4.2. For any $\Lambda \in \mathbf{C}$ and $X>0$, the unique solution of the initial value problem

$$
\left\{\begin{array}{l}
y^{\prime \prime}-\left(\frac{\Lambda^{2}}{x}+a^{2}\right) y=0 \quad \text { in }[-X, X] \\
y^{\prime}(0)=1
\end{array}\right.
$$

is given in terms of a confluent hypergeometric function [5, Chapter 13]: $y(x)=x e^{-a x} M\left(1+\Lambda^{2} /(2 a), 2,2 a x\right)$. For this problem, $g(x)=a^{2}$ and, from Theorem 2.3 with $f(x, y)=g(x) y$, we have that, for $n=0,1,2, \ldots$,

the sequence

$$
\left\{\begin{array}{l}
y_{0}(x)=\phi(x):=\frac{\sqrt{x}}{\Lambda} I_{1}(2 \Lambda \sqrt{x}) \\
y_{n+1}(x)=\phi(x)+2 a^{2} \int_{0}^{x} \sqrt{x t}\left[I_{1}(2 \Lambda \sqrt{x}) K_{1}(2 \Lambda \sqrt{t})\right. \\
\left.\quad-K_{1}(2 \Lambda \sqrt{x}) I_{1}(2 \Lambda \sqrt{t})\right] y_{n}(t) d t
\end{array}\right.
$$

converges to the solution $y(x)$ uniformly for $x \in[-X, X]$. Table 2 shows some numerical results for the approximation supplied by the recursion (43) compared with the approximation supplied by Olver's algorithm. 


\begin{tabular}{c|c|c|c|}
\hline \multicolumn{4}{|c}{$x=1$} \\
\hline \hline$\Lambda$ & $n$ & Olver's method & Formula (42) \\
\hline & 1 & 0.48174856 & 0.00012789 \\
-0.5 & 3 & 0.14351156 & $2.68 e-10$ \\
& 5 & 35.44078569 & $3.80 e-16$ \\
\hline \multirow{3}{*}{1} & 1 & 0.02009256 & 0.00027836 \\
& 3 & 0.00093302 & $6.29 e-10$ \\
& 5 & 0.00178991 & $2.83 e-15$ \\
\hline & 1 & 0.00057733 & 0.00005885 \\
$-5^{1 / 3}$ & 3 & $1.12 e-6$ & $1.8 e-10$ \\
& 5 & $8.22 e-8$ & $2.6 e-15$ \\
\hline & 1 & 0.00009711 & 0.00001251 \\
$-10^{1 / 3}$ & 3 & $4.6 e-8$ & $8.18 e-11$ \\
& 5 & $8.6 e-10$ & $4.3 e-14$ \\
\hline \hline
\end{tabular}

$x=-1$

\begin{tabular}{|c|c|c|c|}
\hline \hline$\Lambda$ & $n$ & Olver's method & Formula (42) \\
\hline \multirow{3}{*}{0.5} & 1 & 0.0246796 & 0.0000754845 \\
& 3 & 0.0123801 & $1.4 e-10$ \\
& 5 & 11.441990 & $1.4 e-16$ \\
\hline \multirow{3}{*}{1} & 1 & 0.00172736 & 0.0000770293 \\
& 3 & 0.0000585129 & $1.4 e-10$ \\
& 5 & 0.000155435 & $9.3 e-16$ \\
\hline \multirow{3}{*}{$5^{1 / 3}$} & 1 & 0.0000200375 & 0.000107348 \\
& 3 & $1.4 e-7$ & $2.1 e-10$ \\
& 5 & $3.2 e-9$ & $1.9 e-16$ \\
\hline \multirow{3}{*}{$10^{1 / 3}$} & 1 & 0.0000820701 & 0.000276735 \\
& 3 & $9.4 e-8$ & $5.8 e-10$ \\
& 5 & $7.0 e-10$ & $1.7 e-14$ \\
\hline
\end{tabular}

TABLE 1. Numerical experiments about the relative errors in the approximation of the solution of problem given in Example 2 using Olver's method and formula (42) for different values of $\Lambda$ and $n$.

5. Final remarks. In Theorem 2.1 we have proposed a kind of Picard-Lindelöf's iteration, recurrence (13), to approximate, uniformly for $x \in[-X, X]$, the unique solution of problem (10). When $\Lambda$ is large compared with the Lipschitz constant $K$ of $f(x, y)$, (13) converges faster than the standard Picard-Lindelöf's iteration. As Olver's expansion, the expansion (13) is asymptotic for large $\Lambda$ but has the advantage of 


\begin{tabular}{c|c|c|c|}
\hline \multicolumn{4}{|c}{$x=0.05$} \\
\hline \hline$\Lambda$ & $n$ & Olver's method & Formula (43) \\
\hline \multirow{3}{*}{0.5} & 2 & $1 . e-4$ & $8.3 \mathrm{e}-7$ \\
& 3 & 0.011 & $1.97 e-10$ \\
& 4 & 0.016 & $9.4 e-13$ \\
\hline \multirow{3}{*}{1} & 2 & $6.25 e-6$ & $8.22 \mathrm{e}-7$ \\
& 3 & 0.00016 & $1.95 e-10$ \\
& 4 & $6.3 e-5$ & $4.6 e-13$ \\
\hline & 2 & $7.15 e-7$ & $8 . e-7$ \\
$-5^{1 / 3}$ & 3 & $6.5 e-6$ & $1.9 e-10$ \\
& 4 & $8.6 e-7$ & $1.4 e-12$ \\
\hline & 2 & $2.8 e-7$ & $7.9 e-7$ \\
$-10^{1 / 3}$ & 3 & $1.6 e-6$ & $1.85 e-10$ \\
& 4 & $1.35 e-7$ & $2.2 e-12$ \\
\hline
\end{tabular}

\begin{tabular}{c|c|c|c|}
\hline \multicolumn{4}{c}{$x=-0.1$} \\
\hline \hline$\Lambda$ & $n$ & Olver's method & Formula (43) \\
\hline \multirow{3}{*}{0.5} & 2 & $1.8 \mathrm{e}-4$ & $1.3 \mathrm{e}-5$ \\
& 3 & $9.3 \mathrm{e}-3$ & $1.27 e-8$ \\
& 4 & 0.09 & $1.47 e-11$ \\
\hline \multirow{3}{*}{1} & 2 & $3.5 \mathrm{e}-5$ & $1.4 \mathrm{e}-5$ \\
& 3 & $1.2 \mathrm{e}-4$ & $1.3 e-8$ \\
& 4 & $3.7 \mathrm{e}-4$ & $1.1 e-11$ \\
\hline \multirow{3}{*}{$5^{1 / 3}$} & 2 & $2.9 \mathrm{e}-5$ & $1.4 \mathrm{e}-5$ \\
& 3 & $2 . \mathrm{e}-5$ & $1.38 e-8$ \\
& 4 & $3.3 \mathrm{e}-5$ & $1.9 e-11$ \\
\hline \multirow{3}{*}{$10^{1 / 3}$} & 2 & $3 . \mathrm{e}-5$ & $1.5 \mathrm{e}-5$ \\
& 3 & $2.8 \mathrm{e}-5$ & $1.45 e-8$ \\
& 4 & $3 . \mathrm{e}-5$ & $1 . e-11$ \\
\hline
\end{tabular}

TABLE 2. Numerical experiments about the relative errors in the approximation of the solution of problem given in Example 3 using Olver's method and formula (43) for different values of $\Lambda$ and $n$.

also being convergent for any $\Lambda \in \mathbf{C}, \Re \Lambda \geq 0$. A formal difference between both expansions is that Olver's expansion is of Poincaré type, whereas expansion (13) is not. An important difference is that this method can be used for nonlinear problems with Lipchitz continuous functions $f(x, y)$, whereas Olver's method requires a linear problem with an analytic function $g$. On the other hand, this method is valid 
for bounded domains, whereas Olver's method is valid for unbounded domains.

The existence and uniqueness of the solution of (26) is a new result, since Picard-Lindelöf's theorem does not apply. Then, Theorem 2.3 is a kind of Picard-Lindelöf's theorem for second order differential equations with initial datum given at the regular singular point of the equation. It is worth noting that, as a difference from the regular problem, only one initial datum is required: the value of the derivative at the regular singular point.

A different asymptotic as well as convergent method is given in [2]. As a difference from the method presented here, it is valid for unbounded domains of $x$. On the other hand, it requires a more stringent condition for the function $g(x)$ : it must be analytic and posses a certain asymptotic behavior at infinity.

Acknowledgments. The authors thank the referees for their careful reading of the manuscript and valuable comments.

\section{REFERENCES}

1. P.B. Bailey, L.F. Shampine and P.E. Waltman, Nonlinear two point boundary value problems, Academic Press, New York, 1968.

2. T.M. Dunster, Convergent expansions for the solutions of linear ordinary differential equations having a simple turning point, with an application to Bessel function, Stud. Appl. Math. 107 (2001), 293-323.

3. W.G. Kelley and A.C. Peterson, The theory of differential equations, John Wiley \& Sons, New York, 1962.

4. J.L. López, Olver's asymptotic method revisited, Case I, J. Math. Anal. Appl., accepted.

5. NIST Digital Library of Mathematical Functions, http://dlmf .nist.gov/.

6. F.W.J. Olver, Asymptotics and special functions, Academic Press, New York, 1974.

7. R.B. Paris, On the use of Hadamard expansions in hyperasymptotic evaluation of Laplace-type integrals, I. Real variable, J. Comp. Appl. Math. 167 (2004), 293-319.

8. $\quad$ On the use of Hadamard expansions in hyperasymptotic evaluation of Laplace-type integrals, II. Complex variable, J. Comp. Appl. Math. 167 (2004), 321-343.

9. , Hadamard expansions and hyperasymptotic evaluation. An extension of the method of steepest descents, Encycl. Math. Appl. 141, Cambridge University Press, Cambridge, 2011. 
10. I. Stackgold, Green's functions and boundary value problems, John Wiley \& Sons, New York, 1998.

11. N.M. Temme, Special functions: An introduction to the classical functions of the mathematical physics, John Wiley \& Sons, New York, 1989.

12. F. Verhulst, Nonlinear differential equations and dynamical systems, Springer-Verlag, New York, 1996.

13. R. Wong, Asymptotic approximations of integrals, Academic Press, New York, 1989.

Dpt. de Matemática Aplicada, iUma, Universidad de Zaragoza, Zaragoza, SPAIN

Email address: cferrei@unizar.es

Dpt. de Ingeniería Matemática e Informática, Universidad Pública de Navarra and BIFI, Zaragoza, Zaragoza, Spain

Email address: jl.lopez@unavarra.es

Dpt. de Matemática Aplicada, iUma, Universidad de Zaragoza, Zaragoza, SPAIN

Email address: ester.perez@unizar.es 